\title{
Note on highly connected monochromatic subgraphs in 2-colored complete graphs
}

\author{
Shinya Fujita* Colton Magnant ${ }^{\dagger}$ \\ Submitted: Jul 25, 2009; Accepted: Nov 13, 2009; Published: Jan 12, 2011 \\ Mathematics Subject Classification: 05C15, 05C40
}

\begin{abstract}
In this note, we improve upon some recent results concerning the existence of large monochromatic, highly connected subgraphs in a 2-coloring of a complete graph. In particular, we show that if $n \geq 6.5(k-1)$, then in any 2-coloring of the edges of $K_{n}$, there exists a monochromatic $k$-connected subgraph of order at least $n-2(k-1)$. Our result improves upon several recent results by a variety of authors.
\end{abstract}

\section{Introduction}

It is easy to see that for any graph $G$, either $G$ or its complement is connected. This is equivalent to saying there exists a connected color in any 2-coloring of $K_{n}$. However, when we try to find a subgraph with higher connectivity, we cannot hope to find such a spanning subgraph. In order to see this, consider the following example. All standard notation comes from [3].

Consider the following example from [1]. Let $G_{n}=H_{1} \cup \cdots \cup H_{5}$ where $H_{i}$ is a red complete graph $K_{k-1}$ for $i \leq 4$ and $H_{5}$ is a red $K_{n-4(k-1)}$ where $n>4(k-1)$. To this structure, we add all possible red edges between $H_{5}, H_{1}$ and $H_{2}$ and from $H_{1}$ to $H_{3}$ and from $H_{2}$ to $H_{4}$. All edges not already colored in red are colored in blue. In either color, there is no $k$-connected subgraph of order larger than $n-2(k-1)$.

Since a spanning monochromatic subgraph is more than we could hope for, we consider finding a highly connected subgraph that is as large as possible. Along this line, Bollobás and Gyárfás proposed the following conjecture.

Conjecture 1 ([1]) For $n>4(k-1)$, every 2-coloring of $K_{n}$ contains a $k$-connected monochromatic subgraph with at least $n-2(k-1)$ vertices.

*Department of Mathematics, Gunma National College of Technology. 580 Toriba, Maebashi, Gunma, Japan 371-8530. shinya.fujita.ph.d@gmail.com Supported by JSPS Grant No. 20740068

${ }^{\dagger}$ Department of Mathematics, Lehigh University, 27 Memorial Dr, W. Bethlehem, PA, USA, 18015. dr.colton.magnant@gmail.com 
In order to see that the bound on $n$ is the best possible, consider the example $G_{n}$ above with $n=4(k-1)$ (so $H_{5}=\emptyset$ ). In [1], the authors showed that this conjecture is true for $k \leq 2$. Also, in [4], Liu, Morris and Prince showed the conjecture holds for $k=3$, but for other cases, it remains open. As a weaker result, in [6] the authors proved the following.

Theorem 1 ([6]) If $n \geq 13 k-15$ then every 2 -coloring of $K_{n}$ contains a monochromatic $k$-connected subgraph of order at least $n-2(k-1)$.

In a related result, Bollobás and Gyárfás also proved the following.

Theorem 2 ([1]) If Conjecture 1 holds for $4(k-1)<n<7(k-1)$ then Conjecture 1 is true.

In this note, we improve both of these results as follows:

Theorem 3 If $n>6.5(k-1)$ then any 2-coloring of $K_{n}$ contains a monochromatic $k$ connected subgraph of order at least $n-2(k-1)$.

By improving the constant from 13 to 6.5 , we also slightly improve other results from [5] in some cases. As these improvements are very minor, we omit details. Since any $k$ connected graph has the minimum degree at least $k$, we immediately obtain the following corollary.

Corollary 4 If $n>6.5(k-1)$, then any 2-coloring of $K_{n}$ contains a monochromatic subgraph of order at least $n-2(k-1)$ with the minimum degree at least $k$.

This corollary slightly improves a result in [2], which deals with the monochromatic large subgraph with a specified minimum degree in general graphs. When we focus on complete graphs, their work shows that the conclusion holds if $n \geq 7 k+4$.

\section{Proof of Theorem 3}

Consider a 2-coloring $G$ of $K_{n}$ with the colors red and blue. The proof proceeds by induction on $k$. The cases for $k \leq 2$ follow from [1] and the case $k=3$ follows from [4] but we will not need this assuption so we simply suppose $k \geq 3$. By induction, there exists a $(k-1)$-connected subgraph in one color (suppose red) of order at least $n-2(k-2)$. If this subgraph is $k$-connected, this is a desired subgraph so we may assume the connectivity is exactly $k-1$.

Let $G_{r}$ be the largest $(k-1)$-connected red subgraph and consider a minimum cutset $C$ (of order $k-1$ ) of $G_{r}$. Let $A^{C}$ and $B^{C}$ be a bipartition of the vertices of $G_{r} \backslash C$ such that $A^{C}$ (and likewise $B^{C}$ ) is the union of vertices in components of $G_{r} \backslash C$ and we choose such unions with $\left|A^{C}\right| \geq\left|B^{C}\right|$ and $\left|B^{C}\right|$ maximum. Choose such a cutset $C$ so that $\left|B^{C}\right|$ 
is maximized and define $A^{\prime}=A^{C}$ and $B^{\prime}=B^{C}$. By definition, all edges between $A^{\prime}$ and $B^{\prime}$ are blue. This forms a complete bipartite graph in blue. Define $D^{\prime}=G \backslash G_{r}$.

First suppose $\left|B^{\prime}\right| \geq k$, which implies that this blue complete bipartite graph is $k$ connected. Note that $\left|D^{\prime}\right| \leq 2(k-2)$ and, since $\left|G_{r}\right|$ is maximum, every vertex in $D^{\prime}$ has at most $k-2$ red edges to $G_{r}$. This means that each vertex of $D^{\prime}$ must have at least $\left|G_{r}\right|-(k-2)$ blue edges to $G_{r}$. More specifically, each vertex must have at least $\left|A^{\prime} \cup B^{\prime}\right|-(k-2)>k$ blue edges to $A^{\prime} \cup B^{\prime}$ (since $\left.n \geq 5 k-7\right)$. This means that $A^{\prime} \cup B^{\prime} \cup D^{\prime}$ induces a blue $k$-connected graph of order exactly $n-(k-1)$, thus proving the theorem in this case.

Hence, we assume $\left|B^{\prime}\right|<k$. Let $B$ be the set of vertices satisfying the following conditions:

1. $|B|$ is maximum subject to $|B|<3(k-1)$.

2. Each vertex of $B$ has at most $k-1$ red edges to $G \backslash B$.

Certainly such a set $B$ exists since both $D^{\prime}$ and $D^{\prime} \cup B^{\prime}$ satisfy Property 2 and we know that $\left|D^{\prime}\right| \leq 2(k-1)$ and $\left|B^{\prime}\right| \geq 1$.

Claim $1|B| \geq 2(k-1)$.

Proof of Claim 1: $\quad$ Suppose $|B|<2(k-1)$ and consider the graph $G_{r}$ induced on the red edges in $G \backslash B$. If this graph is $k$-connected, it would be a desired subgraph so we know $\kappa\left(G_{r}\right) \leq k-1$. As above, if there exists a cutset $C^{\prime}$ of $G_{r}$ and a partition of the components of $G_{r} \backslash C^{\prime}$ so that each part has order at least $k$, then we could find a $k$-connected blue subgraph of order at least $n-\left|C^{\prime}\right| \geq n-(k-1)$ which would again be a desired subgraph (note that each vertex of $B$ has at least $k$ blue edges to $G_{r}$ ). Hence, there exists a cutset $C^{\prime}$ of order $\left|C^{\prime}\right| \leq k-1$ and a set of vertices $B^{*}$ (think of a component of $G_{b} \backslash C^{\prime}$ ) of order $\left|B^{*}\right| \leq k-1$ which have red edges only to $C^{\prime}$ in $G_{r}$. The set $B \cup B^{*}$ forms a set larger than $B$ satisfying Properties 1 and 2, a contradiction.

$\square_{\text {Claim } 1}$

Let $A=G \backslash B$ and consider the blue bipartite graph $G_{b}$ induced on $A \cup B$. Since we have assumed $n>6.5(k-1)$, we see that $|A| \geq 3.5(k-1)+1$. At this point, it is worth while to note that, by Lemma 10 in [5], Theorem 3 holds for $n>8(k-1)$. Part of what remains of our proof is a strengthening of the ideas presented in [5].

We now claim that there exists a large $k$-connected subgraph of $G_{b}$ which serves as a desired structure. Hence, we restrict our attention to $G_{b}$. Assume $G_{b}$ is not $k$-connected. Consider a minimum cutset $C$ with $|C| \leq k-1$.

Claim $2 C \subseteq B$.

Proof of Claim 2: In order to prove this claim, it suffices to show that a cutset of order at most $k-1$ cannot separate two vertices of $B$. This would imply that any cutset including vertices of $A$ is not minimal and hence, complete the proof. 
Each vertex of $B$ has at least $|A|-(k-1)$ edges to $A$ which means that each pair of vertices in $B$ shares at least $|A|-2(k-1) \geq k$ common neighbors (note that this requires only $n>6(k-1))$. Hence, no pair of vertices in $B$ can be separated by a cutset of order at most $k-1$, thereby proving the claim.

$\square_{\text {Claim } 2}$

Since $G_{b}$ is bipartite, every component of $G_{b} \backslash C$ which does not contain a vertex of $B$ is a single vertex (in $A$ ). Hence, each of these vertices has degree at most $|C| \leq k-1$. Let $A^{*}$ be the vertices $v \in A$ with $d_{b}(v) \leq k-1$. Our first goal is to show that $\left|A^{*}\right|=t \leq 2(k-1)$. From the definitions, there are at most $t(k-1)+|B|(|A|-t)$ blue edges between $A$ and $B$. Conversely, recall that there are at least $|B|(|A|-(k-1))$ edges between $A$ and $B$ since each vertex of $B$ has many blue edges to $A$. This means

$$
t(k-1)+|B|(|A|-t) \geq|B|(|A|-(k-1))
$$

which, using the fact that $|B| \geq 2(k-1)$, implies

$$
t \leq \frac{|B|(k-1)}{|B|-(k-1)} \leq 2(k-1)
$$

as required.

Let $A^{\prime \prime}=A \backslash A^{*}$ and let $G_{b}^{\prime \prime}=B \cup A^{\prime \prime}=G_{b} \backslash A^{*}$ (the graph remaining after the removal of the above singleton vertices). We would now like to show that $G_{b}^{\prime \prime}$, which has order $n-t \geq n-2(k-1)$, is $k$-connected. Let $C^{\prime \prime}$ be a minimum cutset of $G_{b}^{\prime \prime}$ and suppose $\left|C^{\prime \prime}\right| \leq k-1$. Let $t^{\prime}$ be the maximum red degree from vertices in $B \backslash C^{\prime \prime}$ into $A^{*}$. From this we get the following inequalities

$$
t(|B|-(k-1)) \leq e_{r}\left(B, A^{*}\right) \leq t^{\prime}\left|B \backslash C^{\prime \prime}\right|+t\left|B \cap C^{\prime \prime}\right|
$$

which implies

$$
t^{\prime} \geq t-\frac{t(k-1)}{\left|B \backslash C^{\prime \prime}\right|}
$$

We would now like to show that $\left|A^{\prime \prime} \backslash C^{\prime \prime}\right| \leq 2(k-1)-t^{\prime}$. In order to accomplish this task, let $X$ and $Y$ be the two components (or collections of components) of $G_{b}^{\prime \prime} \backslash C^{\prime \prime}$ and choose a vertex $v \in B \backslash C^{\prime \prime}$ such that $e_{r}\left(v, A^{*}\right)=t^{\prime}$. Notice that, by the definition of $A^{*}$ and $G_{b}^{\prime \prime}=G_{b} \backslash A^{*}$, we know that $B \cap X$ and $B \cap Y$ are both nonempty. Without loss of generality, suppose $v \in B \cap X$. Since all edges from $v$ to $A^{\prime \prime} \cap Y$ are red, we know that $\left|A^{\prime \prime} \cap Y\right| \leq k-1-t^{\prime}$. Now let $v^{\prime}$ be a vertex in $B \cap Y$. Since all edges from $v^{\prime}$ to $A^{\prime \prime} \cap X$ are red, we get $\left|A^{\prime \prime} \cap X\right| \leq k-1$. These two bounds show that $\left|A^{\prime \prime} \backslash C^{\prime \prime}\right| \leq 2(k-1)-t^{\prime}$.

Using (1) and (2), this implies

$$
\begin{aligned}
n & =\left|C^{\prime \prime}\right|+\left|A^{\prime \prime} \backslash C^{\prime \prime}\right|+\left|B \backslash C^{\prime \prime}\right|+t \\
& \leq\left|C^{\prime \prime}\right|+2(k-1)-t^{\prime}+\left|B \backslash C^{\prime \prime}\right|+t \\
& \leq(k-1)+2(k-1)-\left(t-\frac{t(k-1)}{\left|B \backslash C^{\prime \prime}\right|}\right)+\left|B \backslash C^{\prime \prime}\right|+t \\
& \leq 3(k-1)+\left|B \backslash C^{\prime \prime}\right|+\frac{|B|(k-1)^{2}}{[|B|-(k-1)]\left|B \backslash C^{\prime \prime}\right|} .
\end{aligned}
$$


Hence, we need only show that

Fact 1

$$
\left|B \backslash C^{\prime \prime}\right|+\frac{|B|(k-1)^{2}}{[|B|-(k-1)]\left|B \backslash C^{\prime \prime}\right|} \leq 3.5(k-1) .
$$

Proof: In order to prove this fact, we maximize the left hand side (LHS) over the values $2(k-1) \leq|B| \leq 3(k-1)$ and $(k-1) \leq\left|B \backslash C^{\prime \prime}\right| \leq 3(k-1)$ (also certainly $\left.|B| \geq\left|B \backslash C^{\prime \prime}\right|\right)$. It is easy to see this maximum occurs at one of the boundary points of our allowed values so we need only check these points. The largest value occurs when $|B|=\left|B \backslash C^{\prime \prime}\right|=3(k-1)$ which yeilds $L H S \leq 3.5(k-1)$.

Fact 1

Hence $n \leq 3(k-1)+3.5(k-1)=6.5(k-1)$ which is a contradiction, completing the proof of Theorem 3 .

Since we actually know $|B|<3(k-1)$, the result in Fact 1 (and hence Theorem 3) may be improved slightly. For the sake of simplicity, this computation is omitted.

Acknowledgement: The authors would like to thank Daniel M. Martin for his help and ideas leading up to the original proof of this result. We would also like to thank the anonymous referee for very helpful comments.

\section{References}

[1] B. Bollobás and A. Gyárfás. Highly connected monochromatic subgraphs. Discrete Mathematics, 308(9):1722-1725, 2008.

[2] Y. Caro and R. Yuster. The order of monochromatic subgraphs with a given minimum degree. Electron. J. Combin., 10:R32, 8 pp., 2003.

[3] G. Chartrand and L. Lesniak. Graphs \& Digraphs. Chapman \& Hall/CRC, Boca Raton, FL, fourth edition, 2005.

[4] H. Liu, R. D. Morris, and N. Prince. Highly connected monochromatic subgraphs: Addendum. Manuscript.

[5] H. Liu, R. D. Morris, and N. Prince. Highly connected multicoloured subgraphs of multicoloured graphs. Discrete Math., 308(22):5096-5121, 2008.

[6] H. Liu, R. D. Morris, and N. Prince. Highly connected monochromatic subgraphs of multicolored graphs. J. Graph Theory, 61(1):22-44, 2009. 